\title{
RESUMEN
}

Como todos los sectores de la sociedad, la pandemia también ha impactado a las Instituciones de Educación Superior (IES). La educación a distancia ha sido implementada de forma acelerada y ausente de planificación como respuesta a la necesidad de no congregar a estudiantes y docentes presencialmente, pero a la vez, poder continuar el proceso formativo. Este trabajo aborda la percepción de docentes de Contador Público y Auditor a través de encuestas aplicadas a 98 docentes en 8 universidades, privadas y públicas. Entre los resultados observamos más coincidencias que diferencias en la percepción la educación a distancia de emergencia, como el mayor esfuerzo docente, problemas en los mecanismos de evaluación y logro de aprendizajes.

Palabras claves: Educación superior, contador público, Chile, Covid-19

\section{PERCEPCIÓN DE LA EDUCACIÓN A DISTANCIA DE EMERGENCIA EN AÑO DE COVID-19 EN CARRERAS DE AUDITORIA DE UNIVERSIDADES CHILENAS.}

\author{
Fernando Morales Parada ${ }_{1}$ / Francisco Nova Rodríguez
}




\title{
PERCEPTION ANALYSIS OF EMERGENCY DISTANCE EDUCATION IN THE TIME OF COVID-19 IN PUBLIC ACCOUNTANT AND AUDITOR OF CHILEAN UNIVERSITIES
}

\begin{abstract}
Equivalent to all sectors of society, the current pandemic has also impacted Higher Education Institutions. Distance education has been implemented in an accelerated way and without planning as a response to the need not to bring together students and teachers in person, but at the same time, as a quick action to be able to continue the training process. This inquiry addresses the perception of Public Accountant and Auditor's teachers through surveys applied to 98 teachers in 8 universities, from the private and public sector. Among the results, we observed more coincidences than differences in the perception of emergency distance education, for instance, higher teaching effort, problems with evaluation mechanisms and learning achievement.
\end{abstract}

Keywords: Higher education, public accountant, Chile, Covid-19 


\section{INTRODUCCIÓN}

En la actualidad se escucha con mayor fuerza el término "educación a distancia", debido a la crisis sanitaria provocada por el Covid-19, sin embargo, este término trae aparejado dificultades que deben considerarse desde diferentes puntos de vistas. En algunos países esta metodología de enseñanza se desarrolla hace muchos años, aunque no de la misma forma. Los avances tecnológicos han permitido introducir nuevas formas de educación a distancia incorporando información en línea gracias al internet y sistemas computacionales (Cookson y Domínguez, 2015). Sin embargo, el desarrollo actual de la experiencia educativa remota busca dar una respuesta temporal a un problema inmediato (Bozkurt y Sharma, 2020), como lo es la crisis sanitaria, también denominado por diversos autores como educación a distancia de emergencia e incluso con el anglicismo corona-teaching (Pedró, 2020; Díaz, 2020; Kolak et al, 2021) o EdTech (Jordan et al, 2021).

Antes de la pandemia por Covid-19, UNESCO (2020) había ya recabado evidencia de los millones de personas que a nivel mundial no recibían educación inclusiva $y$ de calidad en los diferentes niveles de enseñanza, sea por limitaciones significativas en la cobertura del servicio educativo o por el no cumplimiento de estándares de dignidad, justicia y equidad, comprometido en las convenciones suscritas por los propios Estados. Rescatando el valor de UNESCO (2020) en este sentido, Morales et al (2021) busca resaltar que no es suficiente que los docentes posean competencias en TIC y logren transmitírselas a sus estudiantes, de forma adecuada, instándolos a desarrollar verdaderas redes colaborativas que permitan resolver problemáticas de forma más eficientes. Sumado a esto, los trabajos de investigación que aborden un programa específico universitario son escasos aun, existiendo dudas sobre cómo se ha adaptado la formación de los contadores públicos en medio de la nueva modalidad de enanza telemática.

Sin embargo, los factores sociales no quedan ajenos a esta educación improvisada en un principio de la declaración de emergencia sanitaria y que ya gran parte del año académico nos hemos estado adaptando. Como dicen Cristacho, Guerra y Ortega (2008) "América latina es rica en desigualdades, por otro lado, como la incorporación de gran parte de la tecnología se hace sin una base endógena de investigación y desarrollo, la difusión depende de la adopción de tecnologías exógenas, a un ritmo y patrón que varía entre países". En palabras de Quiroz (2020) "Las desigualdades en el acceso a la educación y particularmente en el acceso a la educación de calidad tienen un especial vínculo con la equidad 0 inequidad territorial".

Esa desigualdad de condiciones fue evidente en el inicio de la implementación en el sistema universitario chileno. Estudiantes se movilizaron haciendo paralizaciones en línea, debido a que consideraban que la educación en línea es una medida discriminatoria ya que no todos pueden acceder (Cardiel, 2020), llegando a exigir devolución de sus pagos de matrículas y rebajas de arancel, porque no se están entregando los servicios por los que se está pagando. Sin embargo, 
las universidades reaccionaron rápidamente, por el interés social, pero también de darle viabilidad a sus proyectos educativos y sostenibilidad financiera. Las universidades aportaron de inmediato a sus estudiantes chips para conectividad a internet y equipamientos como aporte directo y tuvieron que invertir en mayores licenciamientos ${ }^{1}$, más servidores o cloud de soporte o más personal para fortalecer equipos docentes-pedagógicos entre otros, a pesar de los impactos negativos en el sistema de educación superior a causa de la alta deserción y morosidad de pago², seguido a los impactos que dejó la crisis social en Chile 2019.

En general, los estudiantes de la región disponen de menos equipamiento que los estudiantes de los países de la OCDE y aunque la mayoría de ellos cuentan con conectividad, todavía existe un grupo considerable de estudiantes que están completamente excluidos, en especial en los países con menos recursos (CEPAL, 2020). Por lo mismo, el sistema chileno registró una importante deserción estudiantil del orden de los 43 mil estudiantes de pregrado y casi 3 mil de postgrado del sistema universitario chileno de acuerdo a los datos que informó MINEDUC (2020). Estos aspectos que son observados desde la mirada amplia del sistema de educación terciario, tendrían impacto desde la particularidad de un programa como el de Contador Público, sin encontrar datos tan específicos en la literatura a la fecha.

Desde la perspectiva interna las universidades quieren y necesitan proporcionar su propio contenido en línea en cada Facultad, Escuela y programa, pero muchos miembros de las comunidades o claustros académicos nunca han diseñado o impartido un curso en línea. Las universidades tuvieron que trabajar con los profesores para tomar decisiones rápidas para adaptar cursos y reinventar seminarios, mejorando la forma en que enseñan en línea (DeVaney et al, 2020). Es justamente en este sentido que nos parece pertinente explorar la realidad de Contador Público en universidades chilenas, que es un programa extendido a lo largo de las universidades chilenas, que forma parte de la segunda área disciplinaria con más matricula en Chile (Administración y Comercio 20,3\% del total matricula al 2021) y ha tenido un fuerte impacto en su matrícula de primer año, en especial tras el inicio de la pandemia administración y comercio la tercera área disciplinar con mayor baja de matrícula (La variación 2017-2021 ha sido de un -11,5\%) según Ios datos SIES (2021). De hecho en las top-20 de matrícula de primer año para el 2021, Contador Público y Auditor se posiciona 15, dando cuenta de su relevancia en el sistema universitario chileno.

Por lo tanto, no cabe duda que la emergencia ha llevado a las instituciones de educación superior a implementar cursos en línea sin mayor preparación, diseño, capacitación 0 evidencia de acuerdo con Cea et al (2020). En este contexto, la tecnología no lo es todo; hay aspectos claves de la calidad del aprendizaje que también se deben considerar, como repensar las prioridades del aprendizaje y garantizar la calidad en la educación en línea. A pesar que las IES (Instituciones de Educación Superior) lo tuvieron más fácil que el sistema educativo escolar según García (2021), ya que muchas universidades ya contaban con plataformas digitales 
que venían utilizando en algunas fases del desarrollo de su docencia, generalmente presencial. Y, en segundo lugar, porque la edad de los estudiantes hace más viable una enseñanza/aprendizaje de este corte, idea confirmada en el estudio realizado por Sapién et al (2020) en la Facultad de Contaduría y Administración de la Universidad Autónoma de Chihuahua en México. De esta forma, parece válido preguntarse cómo ha sido la adaptación, los problemas y desafíos que observan los docentes de un programa como el de contaduría.

La mirada crítica que tienen Murillo y Duk (2020) acerca de que la educación a distancia, tildándola de quimera, ya que es una alternativa sólo para los que tienen equipos de una cierta calidad con acceso a internet en casa, tiene asidero ya que al inicio de la pandemia se evidenció demasiados estudiantes que no contaban con ese recurso de la canasta básica universal según Harari (2018), un computador con internet, ni con las condiciones materiales, ambientales y de espacio para poder beneficiarse de esta opción. Esto genera un nuevo desafío para docentes y alumnos ya que es algo nuevo, el cual tendrán que enfrentar y trabajar en conjunto en post del avance y rendimiento académico positivo. Para los docentes en particular es un gran desafío ya que la modalidad de enseñanza cambia completamente, adaptándola a nuevas metodologías, de enseñanza y nuevas formas de evaluar, las que nos interesa explorar entre docentes de los programas de contaduría en Chile. Esta situación genera un estrés adicional a lo que estamos viviendo como contingencia y aislamiento entre otros factores que van sumando (Palominos y Martínez, 2020) y que queremos comprender desde la perspectiva de profesores de Contador Público y Auditor.

Ahora bien, sin duda la resiliencia y el factor humano está y estará siempre presente, tal como lo abordan Llopiz et al (2020) al señalar que el principal problema que atenta con la educación a distancia es la mentalidad de los actores claves (docentes y alumnos), puesto que existen docentes que no comprenden la necesidad del cambio, por tener arraigado los modelos pedagógicos tradicionales, a lo que agregaríamos que el liderazgo naturalmente viene dado por las IES (Instituciones de Educación Superior) y su cuerpo directivo y docente. En Sangster et al (2020) se incluye una mirada internacional y se reconoce el caso chileno basado en la perspectiva de docentes de la Universidad de Valparaíso, quienes observan en todo este fenómeno la importancia de la revisión de planes y programas académicos, para que los procesos, como la inclusión de metodologías de enseñanza-aprendizaje que utilizan plataformas virtuales se modifiquen eficazmente para lograr resultados de aprendizaje para los estudiantes, así como incorporar procesos más complejos, como el desarrollo de competencias vinculados a la inteligencia emocional, que son indispensables para la creación de entornos propicios para el aprendizaje. Son justamente estos procesos y ajustes los que nos gustaría conocer de mejor forma para Contador Público y Auditor de IES chilenas por la magnitud de estudiantes matriculados en este programa y su importancia social una vez insertos en el mundo del trabajo, en un país donde la fe pública se ha tomado la agenda a partir de diferentes situaciones acontecidas en el país que hace mirar con mayor cuidado a sus profesionales especialista en estas materias y extensible a su proceso formativo. 


\section{REVISIÓN DE LITERATURA}

Los factores sociales y del entorno no quedan ajenos a la capacidad o dificultad de enfrentar la docencia remota que comenzaron a implementar las universidades chilenas, "pues no todos los hogares cuentan con ordenadores adecuados 0 suficientes, muchos tienen un solo ordenador para toda la familia, incluso ninguno, ni con impresoras o escáneres para poder realizar adecuadamente todas las tareas que se pueden exigir. Esta situación se agrava más aún en los hogares donde no hay una formación 0 experiencia previa en la utilización de estas herramientas" (Bardón, 2020).

Ahora bien, Schaffeld y Cordova (2020) observan un elemento crítico que se ha perdido en la nueva modalidad implementada a causa de la emergencia sanitara, al recordar que se supone que el entorno educativo universitario presencial promueve la interacción y la comunicación entre los estudiantes y entre los estudiantes y los profesores, pero la creación y operación de equipos de trabajo en plataformas virtuales se torna una experiencia difícil de abordar para los profesores en tan corto plazo como el que han debido enfrentar; más aún, si se incorporan a este trabajo las redes sociales y los grupos de discusión. Es decir, inevitablemente, la pérdida de contacto social y de las rutinas de socialización que forman parte de la experiencia cotidiana de un estudiante de educación superior tendrán un costo. El aislamiento que va inevitablemente asociado al confinamiento tendrá efectos en términos de equilibrio socioemocional, en particular, en aquellos estudiantes con preexistencias en este sentido. A veces es tan simple, como señalan Varalakshmia y Arunachalamb (2020), "el hogar se considera un lugar de descanso y los alumnos tienen menos conciencia sobre cuándo y cómo estudiar".

Algunas de las tecnologías masivas utilizadas son Skype, Meet, Zoom, Teams entre otras que funcionan como fuentes masivas de aprendizaje y dependen de internet, pero que dejan de lado otro medio popular que también podría servir como educación a distancia, como es la Televisión y la Radio (Laaser y Toloza, 2017). Estos últimos podrían cumplir una función especialmente clave para esa parte de la comunidad escolar y universitaria que reside en sectores rurales 0 incluso urbanos con baja conectividad a internet por la no cobertura. Ahora en el fondo, los medios no deben ser lo predominante, ya que como dicen Palomino y Martínez (2020) "el saber utilizar las tecnologías no es sinónimo de saber enseñar con ellas, menos todavía significa tener mayores capacidades para aprender, pues no basta con saber usar TICs (Tecnologías de Información y Comunicación), si no se sabe enseñar con ellas en el contexto donde los estudiantes deben aprender" lo que pudo quedar de manifiesto al comenzar a usar un método en un medio, para el cual no hubo planificación previa y la adaptación fue en el desarrollo mismo de la enseñanza remota. Aspecto que debiera irse corrigiendo en la medida que avanzan los cursos 0 incluso cambiando de año académico/escolar, ya el 2021 no augura ser del todo ajeno al problema de la pandemia. Es decir, compartimos la idea de Kyong-Jee Kim and Curtis J. Bonk (2006) "la educación online es el futuro de todos los estudiantes" por ende es necesario mejorar el acceso en todo el país. 
Algunos creen que el movimiento no planificado y rápido hacia el aprendizaje en línea, sin capacitación, ancho de banda insuficiente y poca preparación, dará como resultado una experiencia de usuario deficiente que no es propicia para un crecimiento sostenido (Li y Lalani, 2020), mientras que otros como García (2021) y Pedró (2020) creen que surgirá un nuevo modelo híbrido de educación, con importantes beneficios, como el mayor alcance de cobertura, movilidad, mejorar doble presencia con otras actividades laborales sociales y nuevas habilidades necesarias para el mundo del trabajo. "Si la tecnología de aprendizaje en línea puede jugar un papel aquí, nos corresponde a todos explorar todo su potencial"3.

Esta pandemia mundial sin duda ha sido una oportunidad para re-iniciar las inversiones para desarrollar una capacidad adecuada de educación a distancia que esté preparada para la próxima crisis (Manukyan, 2020). Esta mirada creemos que es la correcta, una oportunidad para recordarnos las habilidades que los estudiantes necesitan justo en crisis como estas, como la toma de decisiones informada, resolución creativa de problemas y sobre todo adaptabilidad. Para garantizar que esas habilidades sigan siendo una prioridad para todos los alumnos, la resiliencia también debe integrarse en nuestros sistemas educativos (Estrada, 2020).

Todos los involucrados en esta abrupta migración al aprendizaje en línea deben darse cuenta de que estas crisis y desastres también crean interrupciones en la vida de los estudiantes, los docentes y sus familias en general, fuera de su interacción con la universidad señalan Abreu (2020), por lo que creemos que todos debemos entender las situaciones de cada uno, por el cambio que provocó la pandemia del Covid-19 trasformando el método sumado a una serie de dificultades sociales y personales. Algo así como un ejercicio adicional de empatía en este contexto social de la pandemia.

Por lo tanto, el tránsito a la educación a distancia de emergencia en medio de esta pandemia ha planteado nuevos desafíos al modelo neoliberal y el rol que tiene el Estado para apoyar a las familias más afectadas según Mazzacuto (2020). Ya que a pesar de la ayuda social y económica que se ha sustentado en subsidios y endeudamiento según el autor, esto no ha sido suficiente y la crítica que se instaló masivamente en la ciudadanía a partir del estallido social de octubre del 2019 se ha agudizado, exponiendo con mayor ímpetu los defectos de la estructura capitalista.

Algo mucho más pragmático, ha sido abordar las evaluaciones en cada asignatura en el sistema universitario. Se considera un mínimo necesario que las evaluaciones programadas por el docente sean subidas y realizadas a través las plataformas que ocupa la institución ya que esta es conocida por las dos partes y es lo más semejante a lo que hubiera sido la planificación presencial según García et al (2020). Sin embargo, se discute que implementar evaluaciones que cumplan con criterios de confiabilidad, validez, objetividad y autenticidad (Quesada, 2019) no se presentan con tanta fuerza debido a la poca experiencia y tiempo que se ha tenido para instalar el método. Según García et al (2020), apoyados en diversos 
autores, en situaciones de pocos estudiantes y con modelos muy centrados en la interacción profesor-estudiante los modelos basados en la evaluación continua son perfectamente abordables y admisibles. Por su parte, el profesorado igual lo resiente, según Niño et al (2021) ya que diversas investigaciones coinciden al señalar que cuando los profesores incursionan por primera vez en ambientes educativos en línea experimentan una sensación de agobio y dificultad para impartir sus clases, sobre todo, por la carga de trabajo y tiempo que invierten para planificar actividades, resolver dudas, revisar trabajos y retroalimentar a sus estudiantes.

Bajo este escenario, este estudio tiene por objetivo evaluar la percepción de docentes universitarios vinculados al programa de Contador Público y/o Auditor de una muestra de universidades chilenas, como una contribución a la reflexión con una mirada local sobre del fenómeno de la educación a distancia originada por la irrupción de la pandemia del Covid-19 el año 2020.

\section{MÉTODO Y DISEÑO DE LA INVESTIGACIÓN}

Aunque en Chile las corporaciones educacionales universitarias presentan diferencias en su estructura legal, su dispersión geográfica, tamaño y fuentes de financiamiento, poseen similitudes en la duración de la carrera, número de estudiantes, acreditaciones por la CNA (actualmente ya no hay sistema de acreditación para esta carrera según Ley 21.091) y por lo mismo número de docentes asociados a la carrera.

A causa de esto, hemos seleccionado por conveniencia 8 universidades que imparten la carrera de Contaduría y hemos obtenido 101 cuestionarios de respuestas de docentes del programa como una forma de tener una aproximación a lo que ha sido la educación a distancia en este año de pandemia, sin pretender inferir los resultados a todo el sistema nacional, es una muestra no probabilística que nos permite comprender de forma empírica el fenómeno. Los encuestados se agrupan en 59 pertenecientes a programas de universidades que integran el CRUCH (10 U. de Valparaíso, 9 U. de Concepción, 11 U. Católica del Maule, 10 U. de La Serena, 19 U. del Bío-Bío) y 42 de universidad privadas no CRUCH (7 U. Andrés Bello, 12 U. Finis Terrae, 23 U. Santo Tomás).

El enfoque del estudio es exploratorio basado en la percepción de docentes que imparten clases en la carrera o programa de Contador Público y/o Auditor. El instrumento es un cuestionario digital aplicado en el mes de junio de 2020. Los métodos cuantitativos utilizados son propios de la estadística descriptiva y aplicamos un test de comparación de medianas, para identificar la existencia o no de igualdad entre las respuestas recogidas en estas universidades, aunque en este trabajo priorizamos lo cualitativo. A continuación, en la Tabla 1 se presentan las secciones contenidas en la encuesta aplicada y la fuente para la elaboración de las preguntas realizadas: 
Tabla 1: Secciones del cuestionario y fuente para la elaboración de preguntas

\begin{tabular}{|l|l|}
\hline \multicolumn{1}{|c|}{ Secciones del cuestionario } & \multicolumn{1}{c|}{ Fuente } \\
\hline $\begin{array}{l}\text { Acerca de las estrategias de entrega del } \\
\text { servicio educativo }\end{array}$ & \\
\hline $\begin{array}{l}\text { Cultura digital, innovación académica y } \\
\text { resistencia al cambio }\end{array}$ & $\begin{array}{l}\text { Elaboración propia y adaptación de preguntas } \\
\text { de cuestionario internacional aplicado por } \\
\text { Asolación Interamericana de Contabilidad } \\
\text { Habilidades docentes }\end{array}$ \\
\cline { 1 - 2 } $\begin{array}{l}\text { Práctica, didáctica y aprendizaje } \\
\text { significativo }\end{array}$ & (E2020) y Fundación Educación 2020). \\
\hline Sistema de evaluación & \\
\hline
\end{tabular}

Fuente: Elaboración propia

Para efectos de validez, el cuestionario utilizó preguntas ya aplicadas en estudios previos de otros autores (preferentemente centros de estudios de diferentes organismos) y como piloto fue aplicado en dos universidades, de forma comparada una pública y una privada. Esto permitió ajustar preguntas y alternativas de respuesta en algunos casos. Parte del resultado fueron publicados y se pueden encontrar como Nova y Morales (2020) en las referencias.

\section{RESULTADOS}

\section{Acerca de las estrategias de entrega del servicio educativo}

En general, dentro del cuerpo docente, existe un amplio consenso en compartir la determinación de los planteles de impartir docencia bajo la modalidad a distancia bajo el escenario actual. De hecho, el $96 \%$ de los encuestados afirmo compartir esta decisión. Del mismo modo, se destaca que las distintas instituciones han provisto a los docentes herramientas y recursos necesarios para impartir clases en la modalidad a distancia. Los docentes y directivos han dado prioridad a la continuidad del servicio educativo, dejando inclusive en segundo plano la posibilidad de suspensión ante eventuales llamados a paro. En concordancia con lo anterior, los docentes han declarado conocer en más de un $89 \%$ el modelo pedagógico tecnológico o digital implementado por su respectiva institución y su nivel de adhesión al mismo supera el $81 \%$.

Tabla 2: Secciones del cuestionario y fuente para la elaboración de preguntas

\begin{tabular}{|c|c|c|c|c|}
\hline Pregunta & Si & No & No sé & Total \\
\hline $\begin{array}{c}\text { Dado el escenario actual, cree usted que ies } \\
\text { adecuada la decisión de impartir la docencia } \\
\text { en la modalidad a distancia? }\end{array}$ & $96 \%$ & $2 \%$ & $2 \%$ & $100 \%$ \\
\hline $\begin{array}{c}\text { ¿La universidad ha provisto a los docentes } \\
\text { de las herramientas y recursos necesarios } \\
\text { para impartir clases en la modalidad a } \\
\text { distancia? }\end{array}$ & $89 \%$ & $11 \%$ & $0 \%$ & $100 \%$ \\
\hline
\end{tabular}




\begin{tabular}{|c|c|c|c|c|}
\hline Pregunta & Si & No & No sé & Total \\
\hline $\begin{array}{c}\text { En caso de llamados a paros virtuales por } \\
\text { estudiantes, icree que el servicio docente } \\
\text { debe seguir proporcionándose de forma } \\
\text { asincrónica, dejando grabadas las clases? }\end{array}$ & $64 \%$ & $26 \%$ & $10 \%$ & $100 \%$ \\
\hline $\begin{array}{c}\text { ¿Conoces el modelo pedagógico tecnológico } \\
0 \text { protocolo digital implementado por la } \\
\text { institución? }\end{array}$ & $89 \%$ & $7 \%$ & $4 \%$ & $100 \%$ \\
\hline $\begin{array}{c}\text { ¿Adhieres o compartes el contenido del } \\
\text { modelo pedagógico tecnológico o digital } \\
\text { implementado por la institución? }\end{array}$ & $81 \%$ & $7 \%$ & $12 \%$ & $100 \%$ \\
\hline
\end{tabular}

Fuente: Elaboración propia

\section{Cultura digital, innovación académica y resistencia al cambio}

Salvo algunas brechas en universidad del Bío-Bío y la Serena, en general, el cuerpo académico de las instituciones consideradas en el estudio, declara sentirse cercano o muy cercano al uso de herramientas tecnológicas para la educación a distancia, llegando a representar el $70 \%$ de la muestra. Sin embargo su respuesta fue un poco mas cauta cuando se les consultó como había sido su proceso de adaptación a la nueva metodología. Sólo un $56 \%$ de los docentes declaró que este proceso había sido fácil o muy fácil. Las herramientas utilizadas prioritariamente para esta gestión son; Classroom, Meet Socrative, Kahoot, Cisco Webex, Teams, Moodle, Adecca, Blackboard y Zoom. La encuesta revela que el uso de estas plataformas por parte de los docentes ya era una realidad antes de la irrupción de la pandemia. Un 72\% de los encuestados declara haberlas usado previamente, aunque probablemente no con el nivel de intensidad y frecuencia que son utilizadas actualmente. Del mismo modo, reconocen que aún en este escenario, sigue siendo posible innovar en metodologías educativas. Sin embargo advierten que esta nueva metodología ha demandado una mayor cantidad de tiempo para la preparación y revisión de las actividades propias de la docencia. Dentro de las razones que los docentes entienden como las principales dificultades para implementar cursos virtuales, un $45 \%$ señala el poco tiempo para el diseño y la implementación.

Tabla 3: Secciones del cuestionario y fuente para la elaboración de preguntas

\begin{tabular}{|c|c|c|c|c|c|c|}
\hline $\begin{array}{c}\text { Pregunta / Categoría de } \\
\text { respuesta }\end{array}$ & $\begin{array}{c}\text { Absolut. } \\
\text { nada de } \\
\text { cercano }\end{array}$ & $\begin{array}{c}\text { Poco } \\
\text { cercano }\end{array}$ & Indiferente & Cercano & $\begin{array}{c}\text { Muy } \\
\text { cercano }\end{array}$ & Total \\
\hline $\begin{array}{c}\text { ¿Cuál era su nivel } \\
\text { de cercanía con las } \\
\text { herramientas tecnológicas } \\
\text { educacionales previo } \\
\text { al estallido social y la } \\
\text { pandemia covid-19? }\end{array}$ & $6 \%$ & $19 \%$ & $5 \%$ & $43 \%$ & $27 \%$ & $100 \%$ \\
\hline
\end{tabular}




\begin{tabular}{|c|c|c|c|c|c|c|}
\hline $\begin{array}{c}\text { Pregunta / Categoría de } \\
\text { respuesta }\end{array}$ & $\begin{array}{c}\text { Muy } \\
\text { difícil }\end{array}$ & Difícil & Indiferente & Fácil & $\begin{array}{c}\text { Muy } \\
\text { fácil }\end{array}$ & Total \\
\hline $\begin{array}{c}\text { ¿Cómo ha sido el proceso } \\
\text { de adaptación de sus } \\
\text { clases a la metodología a } \\
\text { distancia? }\end{array}$ & $2 \%$ & $31 \%$ & $11 \%$ & $41 \%$ & $15 \%$ & $100 \%$ \\
\hline $\begin{array}{c}\text { Pregunta / Categoría de } \\
\text { respuesta }\end{array}$ & Si & No & No sé & Total & & \\
\hline $\begin{array}{c}\text { Es posible innovar en } \\
\text { metodologías educativas } \\
\text { en el contexto de la } \\
\text { educación a distancia }\end{array}$ & $95 \%$ & $1 \%$ & $4 \%$ & $100 \%$ & & \\
\hline $\begin{array}{c}\text { Pregunta / Categoría de } \\
\text { respuesta }\end{array}$ & $\mathbf{S i}$ & No & Total & $100 \%$ & & \\
\hline $\begin{array}{c}\text { ¿Utilizabas plataformas } \\
\text {-digitales- educativas } \\
\text { antes de COVID-19? }\end{array}$ & $72 \%$ & $28 \%$ & 100 & & \\
\hline
\end{tabular}

Fuente: Elaboración propia

\section{Habilidades docentes}

La valoración docente respecto del proceso de clases a distancia, previo al surgimiento de la pandemia, en promedio era de un 2,5 sobre un total de 5,0 . En línea con esto, los docentes también declaran no haber estado adecuadamente capacitados para impartir clases bajo la metodología a distancia en el momento del inicio de la pandemia. A pesar de lo anterior, y haciendo salvedad en las universidades Finis Terrae y del Bío-Bío, un 93\% de los docentes opinan que las instituciones de educación superior han dispuesto suficientes alternativas de capacitación para la realización de clases en modalidad a distancia. En la misma línea, un $74 \%$ de los encuestados reconoce que la capacitación recibida permite garantizar un adecuado nivel de servicios docentes. Esto ha permitido la continuidad del proceso formativo en la educación superior, en un escenario altamente complejo. Finalmente, en este punto es importante destacar que el $94 \%$ de los docentes declara que es posible innovar en metodologías educativas en este tiempo de cuarentena por coronavirus.

Tabla 4: Secciones del cuestionario y fuente para la elaboración de preguntas

\begin{tabular}{|c|c|c|c|c|}
\hline Pregunta & Si & No & No sé & Total \\
\hline $\begin{array}{c}\text { Previo al estallido social y } \\
\text { la pandemia del covid-19, } \\
\text { ise consideraba usted } \\
\text { capacitado para impartir } \\
\text { clase en la modalidad a } \\
\text { distancia? }\end{array}$ & $54 \%$ & $46 \%$ & $0 \%$ & $100 \%$ \\
\hline
\end{tabular}




\begin{tabular}{|c|c|c|c|c|}
\hline Pregunta & Si & No & No sé & Total \\
\hline $\begin{array}{c}\text { La universidad ha } \\
\text { dispuesto alternativas de } \\
\text { capacitación docente para } \\
\text { la realización de clases en } \\
\text { modalidad a distancia. }\end{array}$ & $93 \%$ & $4 \%$ & $4 \%$ & $100 \%$ \\
\hline $\begin{array}{c}\text { Es posible innovar } \\
\text { en metodologías } \\
\text { educativas en este } \\
\text { tiempo de cuarentena por } \\
\text { coronavirus }\end{array}$ & $94 \%$ & $2 \%$ & $4 \%$ & $100 \%$ \\
\hline $\begin{array}{c}\text { Si su respuesta anterior } \\
\text { fue positiva. iLa } \\
\text { capacitación recibida por } \\
\text { la universidad ha sido } \\
\text { suficiente para garantizar } \\
\text { un adecuado nivel de } \\
\text { servicios docentes en la } \\
\text { impartición de clases en } \\
\text { modalidad a distancia?. }\end{array}$ & $74 \%$ & $7 \%$ & $19 \%$ & $100 \%$ \\
\hline
\end{tabular}

Fuente: Elaboración propia

\section{Práctica, didáctica y aprendizaje significativo}

En general, docentes y directivos destacan los beneficios de haber adoptado la determinación de implementar el proceso de clases a distancia. Un 74\% de ello declara adherir a esta medida, y sólo un 10\% afirma no estar de acuerdo. Debido a la premura con que debió iniciarse el proceso de clases a distancia, un 96\% de los docentes reconoce haber tenido que modificar sus respectivas planificaciones didácticas sobre la marcha. Respecto de las herramientas de comunicación (aula virtual, videoconferencia, etc.) disponibles, un $67 \%$ de los encuestados afirman que ellas permiten generar un ambiente favorable para que se produzca el proceso de enseñanza-aprendizaje y adicionalmente fomentan la autonomía del estudiante. Un $71 \%$ de los docentes reconoce haber cambiado sus paradigmas respecto de la educación a distancia y más de un 95\% de ellos reconocen también que el uso de las tecnologías de información y comunicación en el ámbito educativo, promueve la generación de nuevas posibilidades para la enseñanza y el aprendizaje.

Tabla 5: Secciones del cuestionario y fuente para la elaboración de preguntas

\begin{tabular}{|c|c|c|c|c|}
\hline Pregunta & Si & No & No sé & Total \\
\hline $\begin{array}{c}\text { ¿Percibe beneficios y } \\
\text { ventajas en el proceso de } \\
\text { educación a distancia? }\end{array}$ & $79 \%$ & $10 \%$ & $11 \%$ & $100 \%$ \\
\hline
\end{tabular}




\begin{tabular}{|c|c|c|c|c|}
\hline Pregunta & Si & No & No sé & Total \\
\hline $\begin{array}{c}\text { ¿Consideró } \\
\text { modificaciones en la } \\
\text { planificación didáctica de } \\
\text { su curso, para adaptarlo } \\
\text { a la metodología a } \\
\text { distancia? }\end{array}$ & $96 \%$ & $3 \%$ & $1 \%$ & $100 \%$ \\
\hline $\begin{array}{c}\text { Las herramientas } \\
\text { de comunicación } \\
\text { (el aula virtual 0 de } \\
\text { videoconferencia) } \\
\text { disponibles en la }\end{array}$ & & & & \\
$\begin{array}{c}\text { universidad ipermiten } \\
\text { generar un ambiente } \\
\text { favorable para que se } \\
\text { produzca el proceso de } \\
\text { enseñanza-aprendizaje? }\end{array}$ & $67 \%$ & $7 \%$ & $26 \%$ & $100 \%$ \\
\hline $\begin{array}{c}\text { La experiencia práctica } \\
\text { de la metodología de } \\
\text { enseñanza - aprendizaje a } \\
\text { distancia ina modificado } \\
\text { sus paradigmas al } \\
\text { respecto? }\end{array}$ & $71 \%$ & $22 \%$ & $7 \%$ & $100 \%$ \\
\hline $\begin{array}{c}\text { ¿Las actividades } \\
\text { aplicadas en su curso } \\
\text { promueven el aprendizaje } \\
\text { autónomo del estudiante? }\end{array}$ & $81 \%$ & $4 \%$ & $15 \%$ & $100 \%$ \\
\hline $\begin{array}{c}\text { En su opinión, iel uso } \\
\text { de las Tecnologías } \\
\text { de Información y } \\
\text { comunicación en el } \\
\text { ámbito educativo, } \\
\text { promueve la generación } \\
\text { de nuevas posibilidades } \\
\text { para la enseñanza y el } \\
\text { aprendizaje? }\end{array}$ & & & & \\
\hline
\end{tabular}

Fuente: Elaboración propia

\section{Sistema de evaluación}

El proceso de evaluación representa un desafío en sí mismo. Si a eso se agrega la complejidad de realizar el proceso en entornos virtuales, tenemos como resultado un aspecto de la educación que no está completamente resuelto. En general, los docentes declaran que el proceso de evaluación es uno de los aspectos que menor nivel de confianza les genera en este entorno de clases a distancia. Como una manera de subsanar esta situación, han optado por innovar en sus métodos de evaluación. Un 56\% de la muestra declara estar utilizando registros anecdóticos", 0 "portafolios" priorizando evaluar el proceso enseñanza-aprendizaje más que los contenidos aprendidos. 
El 65\% de los docentes afirma que los instrumentos de evaluación utilizados en la metodología a distancia permiten medir el aprendizaje obtenido por los estudiantes. Por su parte, un $67 \%$ de los encuestados opinó que el modelo de evaluación en entornos virtuales presenta limitaciones para evaluar las competencias asociadas al programa de su asignatura. Además, un $56 \%$ de los encuestados manifiestan cierta desconfianza en los resultados entregados por las evaluaciones tomadas a distancia, principalmente por la facilidad de plagios y copias entre estudiantes.

Al parecer hay una idea generalizada que se pierde el desarrollo de habilidades sociales, la retroalimentación de los alumnos, ausencia de feedback si el alumno está aprendiendo y la atención del alumno. No es posible aplicar un instrumento que permita evaluar el aprendizaje de los estudiantes, hay dificultades para trabajar en equipo, sociabilizar, dificultad para retroalimentar y aclarar dudas, dificultad con las copias en las evaluaciones (por el lado de las desventajas). Sin embargo, la mirada favorable nos indica que se potencia el desarrollo de autonomía y autoaprendizaje, protección de la salud de las personas, menores gastos en traslado, consulta permanente de clases grabadas y se amplía la cobertura de la educación (por el lado de las ventajas).

Tabla 6: Secciones del cuestionario y fuente para la elaboración de preguntas

\begin{tabular}{|c|c|c|c|c|}
\hline Pregunta & Si & No & No sé & Total \\
\hline $\begin{array}{c}\text { Los instrumentos de } \\
\text { evaluación utilizados en la } \\
\text { metodología a distancia son } \\
\text { confiables }\end{array}$ & $41 \%$ & $35 \%$ & $24 \%$ & $100 \%$ \\
\hline $\begin{array}{c}\text { Está innovando en sus } \\
\text { métodos de evaluación, } \\
\text { como "registros } \\
\text { anecdóticos", 0 } \\
\text { "portafolios" priorizando } \\
\text { evaluar el proceso } \\
\text { enseñanza-aprendizaje } \\
\text { más que los contenidos } \\
\text { aprendidos }\end{array}$ & $56 \%$ & $33 \%$ & $11 \%$ & $100 \%$ \\
\hline $\begin{array}{c}\text { Los instrumentos de } \\
\text { evaluación utilizados } \\
\text { en la metodología a } \\
\text { distancia permiten medir el } \\
\text { aprendizaje obtenido por los } \\
\text { estudiantes }\end{array}$ & $65 \%$ & $12 \%$ & $23 \%$ & $100 \%$ \\
\hline $\begin{array}{c}\text { iHay limitaciones para } \\
\text { evaluar las competencias } \\
\text { asociadas al programa de } \\
\text { su asignatura? }\end{array}$ & $67 \%$ & $25 \%$ & $8 \%$ & $100 \%$ \\
\hline
\end{tabular}




\begin{tabular}{|c|c|c|c|c|}
\hline Pregunta & Si & No & No sé & Total \\
\hline $\begin{array}{c}\text { ¿Desconfías de las } \\
\text { tecnologías y la facilidad } \\
\text { de plagios y copias entre } \\
\text { estudiantes al realizar } \\
\text { evaluaciones a distancia en } \\
\text { tiempo real? }\end{array}$ & $56 \%$ & $32 \%$ & $12 \%$ & $100 \%$ \\
\hline
\end{tabular}

Fuente: Elaboración propia

\section{CONCLUSIONES}

Este trabajo tiene por finalidad reflexionar acerca del fenómeno de la educación a distancia a causa de emergencias por la pandemia del covid-19 este año 2020 a partir de la percepción de docentes de ocho universidades nacionales. Dentro de la muestra, se incluye tanto a planteles de educación superior públicos como privados, aunque no era parte del objetivo hacer distinción particular en un segmento u otro.

La excepcionalidad del fenómeno lo amerita, como dice Navarro (2020), "esta crisis es una puerta, un punto de inflexión, un momento de cambio, pero nada asegura que los cambios sean profundos y transformadores, mucho menos que vayan a ser beneficiosos". Por lo mismo creemos que toda reflexión, revisión y pronunciamientos respecto del proceso que estamos viviendo en las IES y específicamente en Contador Público y/o Auditor, ya que viene a contribuir a lograr mejoras en la experiencia de la educación a distancia.

La muestra representa una diversidad importante entre instituciones, ubicados en distintas zonas geográficas, incluyendo planteles públicos y privados, adscritos y no adscritos a gratuidad, pertenecientes y no al consejo de rectores, etc. Del mismo modo, existen distintos tipos de acercamiento al modelo de educación a distancia. Eso puede evidenciase en varias de las respuestas recibidas por parte de los docentes. Los docentes pertenecientes a las universidades de La Serena, Andrés Bello y Santo Tomás presentan en general un mayor nivel de adhesión al modelo, respecto de planteles como Finis Terrae o del Bío-Bío. En nuestra opinión, esto es explicado por el nivel de cercanía con el modelo de educación a distancia, dado que hay planteles cuyo plan de estudio vespertino utiliza desde hace algunos años algunas asignaturas en formato semipresencial.

Los resultados obtenidos, respaldan la determinación de los distintos planteles de continuar impartiendo clases bajo la metodología a distancia, por lo menos hasta que la situación sanitaria se encuentre en un mejor escenario. Sin embargo, también creemos que probablemente muchas de las adecuaciones que fueron implementadas como medidas transitorias y de emergencia, terminarán consolidándose como parte del modelo tradicional más allá de la pandemia, ya que, de alguna manera, han demostrado ser un real aporte al proceso de aprendizaje de los estudiantes. 
Creemos que, si bien la experiencia y la riqueza que entrega un modelo de clases presenciales presenta muchísimas ventajas respecto del modelo remoto, en la medida que el tiempo transcurre, y tanto docentes como estudiantes vayan nivelando su manejo y experiencia con la metodología de clases a distancia, la valoración de las mismas irá incrementándose. La posibilidad de acceder a clases grabadas, los repositorios de información, la flexibilidad que ofrece el modelo, etc. Podría terminar cautivando a personas que en un principio se mostraban altamente reticentes.

Destacamos un alto porcentaje de los docentes que reconoce haber tenido que modificar sus respectivas planificaciones didácticas producto de la impartición de clases a distancia. Y de estos un $70 \%$ afirma que las herramientas de comunicación disponibles permiten generar un ambiente favorable para que se produzca el aprendizaje, la innovación y la autonomía. Es decir, es esperanzador el panorama de cara a una extensión de la emergencia sanitaria en el tiempo.

Como era de esperar, el punto más conflictivo en el estudio resultó ser la evaluación. Este proceso por sí mismo reviste una alta complejidad, y en un entorno virtual, esa complejidad se acentúa. En nuestra opinión a la vista de las respuestas de los docentes, el desafío debe ser abordado en primera instancia por el profesorado. La utilización de instrumentos de evaluación tradicionales, que exijan al estudiante repetir conceptos que puede encontrar con facilidad en textos 0 en algún sitio web, no tienen ningún sentido si ellos no están unidos a un contexto de aplicación práctica. Desde ese punto de vista, los docentes deben innovar en nuevas metodologías de evaluación, apuntando a obtener evidencia del aprendizaje a través de un producto desarrollado por el estudiante.

Finalmente, creemos que los docentes en la educación superior en programas de contaduría si bien han presentado algunos problemas en la implementación del modelo de educación a distancia, pareciera ser que terminarán afinando la didáctica y desarrollando las habilidades tecnológicas necesarias para ejercer su rol en la educación a distancia. Sin embargo, creemos que es la evaluación de los aprendizajes y sobre todo de las competencias adquiridas en donde hay aún un desafío pendiente para nuestro sistema de educación superior. 


\section{REFERENCIAS}

AGUILAR, A. L. S., HOWLET, L. C. P., DIEZ, M. D. C. G., \& BELTRÁN, J. L. B. (2020). La Educación superior durante la contingencia sanitaria COVID-19: Uso de las TIC como herramientas de aprendizaje. Caso de estudio: alumnos de la Facultad de Contaduría y Administración. Revista Latina (78), 309-328.

AIC (2020). Encuesta La docencia en tiempos de Covid 19. Aplicada por la Comisión de Educación en el mes de abril de 2020.

ABREU, J. L. (2020). Tiempos de Coronavirus: La Educación en Línea como Respuesta a la Crisis. Revista Daena (International Journal of Good Conscience), 15(1).

BARDÓN, A. Á. (2020). Alumnos sin acceso a la educación a distancia: la pandemia saca a la luz grandes desigualdades. The Conversation. Obtenido de https://theconversation.com/alumnos-sin-acceso-a-la-educaciona-distancia-la-pandemia-saca-a-la-luz-grandes-desigualdades-135889 [28.04.2021]

BOZKURT, A., \& SHARMA, R. C. (2020). Emergency remote teaching in a time of global crisis due to CoronaVirus pandemic. Asian Journal of Distance Education, 15(1), i-vi.

CEA, F., GARCÍA, R., TURRA, H., CHICO, MOYA, B., SANHUEZA, S., MOYA, R. \& VIDAL, W. (08.06.2020), Educación online de emergencia: Hablando a pantallas en negro Recuperado: https://ciperchile.cl/2020/06/08/ educacion-online-de-emergencia-hablando-a-pantallas-en-negro/

CEPAL, N. (2020). La educación en tiempos de la pandemia de COVID-19. https://unesdoc.unesco.org/ark:/48223/pf0000374075?locale =es

COOKSON, P. \& DOMÍNGUEZ, N. (2015). Un cuento de dos países: Educación a distancia de México y Estados Unidos de América. Revista Española de Educación Comparada, №26, 61-96.

CRISTANCHO, C., GUERRA, M., \& ORTEGA, D. (2008). La dimensión joven de la conectividad en América Latina: brechas, contextos y políticas. Pensamiento iberoamericano, №3, 117-135.

DEVANEY, J., SHIMSHON, G., RASCOFF, M., \& MAGGIONCALDA, J. (2020). Higher ed needs a long-term plan for virtual learning. Harvard Business Review. https://hbr.org/2020/05/higher-ed-needs-a-long-term-plan-forvirtual-learning [28.04.2021]

DÍAZ, M. (2020). La docencia en tiemnpos de Covid 19. Conferencia dictada en el 3er encuentro de instituciones formadoras de Contadores en 
Chile, octubre de 2020. Organiza Comisión de Educación del Colegio de Contadores de Chile A.G.

E2020 (2020). Encuesta Educar en tiempos de pandemia, Parte I. Aplicada en abril de 2020 .

ESTRADA, P. (2020): Educación en tiempos de pandemia: COVID-19 y equidad en el aprendizaje. Observatorio de Innovación Educativa. Obtenido de https://observatorio.tec.mx/edu-news/educacion-en-tiempos-depandemia-covid19

GARCÍA, L. (2021). COVID-19 y educación a distancia digital: preconfinamiento, confinamiento y posconfinamiento. RIED. Revista Iberoamericana de Educación a Distancia, 24(1), pp. 09-32.

GARCÍA-PEÑALVO, F. J., CORELL, A., GRANDE, M., ABELLA GARCÍA, V., \& ALMUZARA, C. (2020) La evaluación online en la educación superior en tiempos de la COVID-19= Online Assessment in Higher Education in the Time of COVID-19. Education in the Knowledge Society (EKS), (21).

CARDIEL, H. C. (2020). Educación y pandemia: una visión académica. Presentación IISUE. Educación y pandemia. Una visión académica, México, UNAM, 9p. Disponible en http://www.iisue.unam.mx/nosotros/ covid/educacion-y-pandemia [25.05.2020]

KYONG, K. \& CURTIS J.B. (2006). The Future of Online Teaching and Learning in Higher Education: EDUCAUSE QUARTERLY, 4, 22 - 30.

JORDAN, K., DAVID, R., PHILLIPS, T., \& PELLINI, A. (2021). Education during the COVID-19: crisis Opportunities and constraints of using EdTech in low-income countries. Revista de Educación a Distancia (RED), 21 (65).

KOLAK, A., MARKIĆ, I., HORVAT, Z., KLEMENČIĆ, M., \& STOJANAC, M. (2021). When the Parent Becomes the Teacher-Attitudes on Distance Learning in the Time of Corona-Teaching from Parents' Perspective. TOJET: The Turkish Online Journal of Educational Technology, 20(1).

LAASER, W. \& TOLOZA, E. A. (2017). The changing role of the educational video in higher distance education. The International Review of Research in Open and Distributed Learning, 18(2).

LI, C. \& LALANI, F. (2020). The COVID-19 pandemic has changed education forever. This is how. In World Economic Forum Vol.29.

LLOPIZ, K., ANDREU, N., GONZÁLEZ, R., ALBERCA, N., FUSTERGUILLÉN, D., \& PALACIOS-GARAY, J. (2020). Prácticas educativas inclusivas a través de la educación a distancia. Experiencias en Cuba. Propósitos y Representaciones, 8(2) 
MANUKYAN, B. (2020). Distance Education in Times of Coronavirus. EVN report. Obtenido de https://www.evnreport.com/raw-unfiltered/distanceeducation-in-times-of-coronavirus [28.04.2021]

MAZZACUTO (2020). El capitalismo en su triple crisis. Obtenido de https://www. sinpermiso.info/textos/el-capitalismo-en-su-triple-crisis [28.04.2021]

MINEDUC (2020) Matrícula en educación superior. Informe de Julio, Servicio de Información de Educación Superior (SIES).

MORALES, M. E. , MOLANO, H., CARDONA, D., \& DELGADO, D. (2021). Analítica de la percepción de docentes y estudiantes sobre el uso de metodologías de enseñanza tradicionales e innovadoras en educación superior. Revista GEON (Gestión, Organizaciones y Negocios), 8(1).

MURILLO, F. J., \& DUK, C. (2020). El Covid-19 y las Brechas Educativas. Revista latinoamericana de educación inclusiva, 14(1), 11-13.

NOVA, F. \& MORALES, F. (2020). Discusión sobre la educación a distancia de emergencia en contador público y auditor, percepciones desde una universidad estatal y privada. Horizontes Empresariales, 19(2), 72-86.

PALOMINOS, M., \& MARTínEZ, V. (2020). Covid-19 y las debilidades de la educación a distancia en Chile. Universidad Católica Silva Henríquez. Obtenido de http://comunicaciones.ucsh.cl/opiniones/covid-19-y-lasdebilidades-de-la-educacion-a-distancia-en-chile/ [28.04.2021]

PEDRÓ, F. (2020). COVID-19 y educación superior en América Latina y el Caribe: efectos, impactos y recomendaciones políticas. Análisis Carolina, 36(1), 1-15.

REYES, C. (2020). Pandemia Covid-19 e Inequidad Territorial: El Agravamiento de las Desigualdades Educativas en Chile. Revista Internacional de Educación para la Justicia Social, 9(3).

QUESADA, R. (2019). Evaluación del aprendizaje en la educación a distancia "en línea". Monográfico VI, 1-15.

SCHAFFELD, G. \& CORDOVA, F. (2020, 30 abril). Desafíos para la educación en tiempos de Covid. Universidad autónoma de Chile. https://www. uautonoma.cl/news/desafios-para-la-educacion-en-tiempos-de-covid/ [28.04.2021]

SANGSTER, A., STONER, G., \& FLOOD, B. (2020). Insights into accounting education in a COVID-19 world. Accounting Education, 29(5), 431-562. Incluye un resumen por países, dentro del cuál está Chile, con un aporte de Cuadra, N. y Maldonado, O., de la Universidad de Valparaíso. 
SAPIÉN, A. L., HOWLET, L. C. P., DIEZ, M. D. C. G., \& BELTRÁN, J. L. B. (2020). La Educación superior durante la contingencia sanitaria COVID-19: Uso de las TIC como herramientas de aprendizaje. Caso de estudio: alumnos de la Facultad de Contaduría y Administración. Revista Latina, (78), 309-328.

SIES Servicio de Información de Educación Superior (2021) "Informe 2021 matrícula de pregrado en educación superior", Junio 2021, Superintendencia de Educación Superior. 16p.

UNESCO (2020) COVID-19 y educación superior de los efectos inmediatos al día después. Recuperado: http://www.iesalc.unesco.org/wp-content/ uploads/2020/04/COVID-19-060420-ES-2.pdf [28.04.2021]

UNESCO. (2020). Marco de competencias de los docentes en materia de TIC. UNESCO. Recuperado: https://es.unesco.org/themes/tic-educacion/ marco-competencias-docentes [28.04.2021]

VARALAKSHMI, R., \& ARUNACHALAM, K. (2020). COVID 2019-Role of faculty members to keep mental activeness of students. Asian Journal of Psychiatry, 51.

\section{NOTAS AL FINAL}

1 Suite de Gmail para acceder a Classroom, Teams de Office o Zoom, entre otros productos 2 Véase nota de prensa UdeC https://noticias.udec.cl/dosis-de-realismo-y-presupuesto-eneducacion-superior/ en línea [20.11.2020]

3 Tomado de https://www.weforum.org/agenda/2020/04/coronavirus-education-globalcovid19-online-digital-learning/ en línea [20.11.2020] 